\title{
Data analysis and key science results of LHAASO-WCDA
}

\author{
Min Zha, ${ }^{a, 1, *}$ Chuandong Gao, ${ }^{b, 1}$ Shicong Hu, ${ }^{a, c, 1}$ Shujie Lin, ${ }^{d, 1}$ Zhen Wang, ${ }^{f, 1}$ \\ Guangman Xiang, ${ }^{c, e, 1}$ Zhiguo Yao $^{a, 1}$ and Hao Zhou ${ }^{f, 1}$ \\ ${ }^{a}$ Key Laboratory of Particle Astrophyics, Institute of High Energy Physics, Chinese Academy of \\ Sciences, \\ 100049 Beijing, China \\ ${ }^{b}$ Institute of Frontier and Interdisciplinary Science, Shandong University, 266237 Qingdao, \\ Shandong, China \\ ${ }^{c}$ University of Chinese Academy of Sciences \\ 100049 Beijing, China \\ ${ }^{d}$ School of Physies and Astronomy 83 School of Physics (Guangzhou), Sun Yat-sen University, \\ 519082 Zhuhai, Guangdong, China \\ ${ }^{e}$ Shanghai Astronomical Observatory, Chinese Academy of Sciences, 200030 Shanghai, China \\ ${ }^{f}$ Tsung-Dao Lee Institute 83 School of Physics and Astronomy, Shanghai Jiao Tong University, \\ 200240 Shanghai, China \\ E-mail: zham@ihep.ac.cn
}

The Water Cherenkov Detector Array (WCDA) is an important component of Large High Altitude Air Shower Observatory (LHAASO). The main scientific target of WCDA is continuously surveying the northern sky at the level of few percent of Crab unit. In this proceeding, based on its first year data of WCDA-1 data, the result about the Crab Nebular observation and its energy spectrum measurment have been presented. And with WCDA full array data taking since Mar. 2021, some preliminary results have been reported.

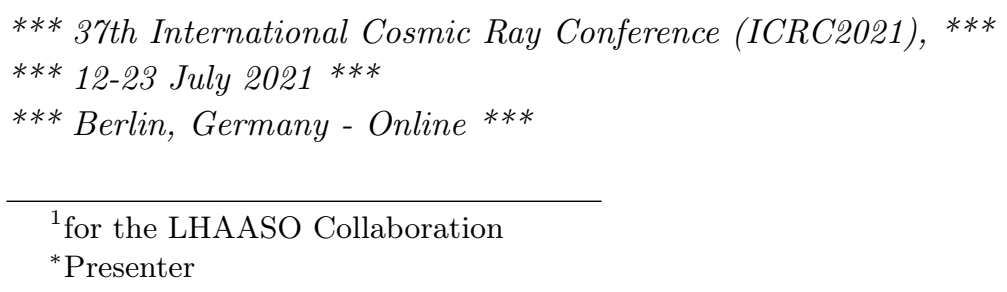



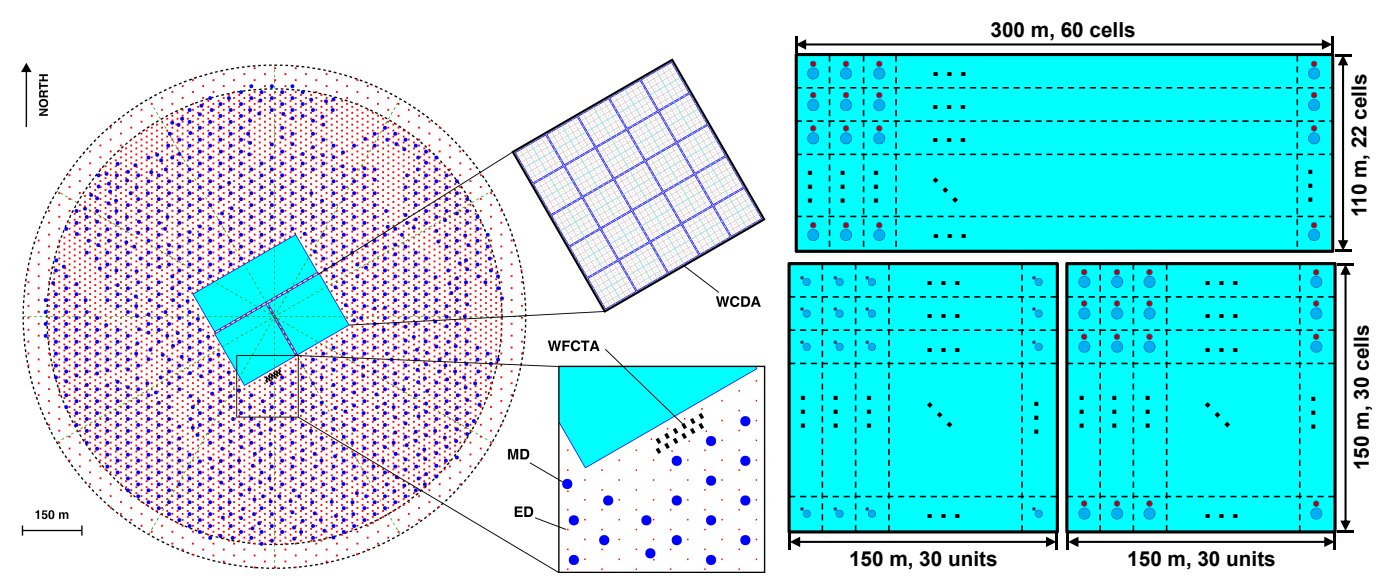

Figure 1: Left panel is the layout of LHAASO. Right panel is the schematic of the WCDA layout. The lower two ponds are WCDA-1 and WCDA-2 from left to right respectively, and the upper one is WCDA-3. The two dots in each detector unit indicate the PMTs, while the dashed lines represent the curtains between units. Two combinations of PMTs are used: 8" and 1.5" PMTs in WCDA-1 and 20" and 3" PMTs in WCDA-2 and WCDA-3.

\section{Introduction}

The Large High Altitude Air Shower Observation(LHAASO) [1, 2] is located in Mountain Haizi with altitude of 4410m asl, ( 29²1'27.6" N, 10008'19.6" E) Daocheng, Sichuan Province, China, which consist of Kilometer-square Array (KM2A), Water Cherenkov Detector Array (WCDA), 18 Wide Field of View Cherenkov Telescope Array (WFCTA). KM2A consists of an extensive air shower detector array covering an area of $1.3 \mathrm{~km}^{2}$ with 5195 scintillator counters (ED, $1 \mathrm{~m}^{2}$ active area) and 1188 muon detectors (MD, water Cherenkov detector with an area of $36 \mathrm{~m}^{2}$ buried under 2.5 meters of dirt).

LHAASO is designed for detection of air showers induced by all kinds of cosmic-ray particles with energy ranging from a few tens of $\mathrm{GeV}$ to a few EeV. Therefore LHAASO is a multi-purpose facility for very-high-energy gamma ray astronomical observation, cosmicray energy spectrum measurements for individual species and other wide-ranging topics associated with the cosmic-rays or air-shower phenomena. For the gamma-ray source survey, the designed sensitivity is about $0.01 \mathrm{CU}$ (Crab Unit). Its wide FoV allows LHAASO to survey $1 / 7$ of the northern sky at any moment for gamma-ray sources. With the operation in full duty cycle, LHAASO scans the entire northern hemisphere every 24 hours. The spectra of all sources in its FoV will be measured with high precision over a wide energy range from $10^{11} \mathrm{eV}$ to $10^{15} \mathrm{eV}$.

\section{WCDA detector array}

The Water Cherenkov Detector Array (WCDA), mainly used for surveying transient phenomena and discovering new sources, which composed of 3 pools, and pool 1 and pool 2 are both $150 \mathrm{~m} \times 150 \mathrm{~m}$, the third pool array is $300 \mathrm{~m} \times 110 \mathrm{~m}$. 

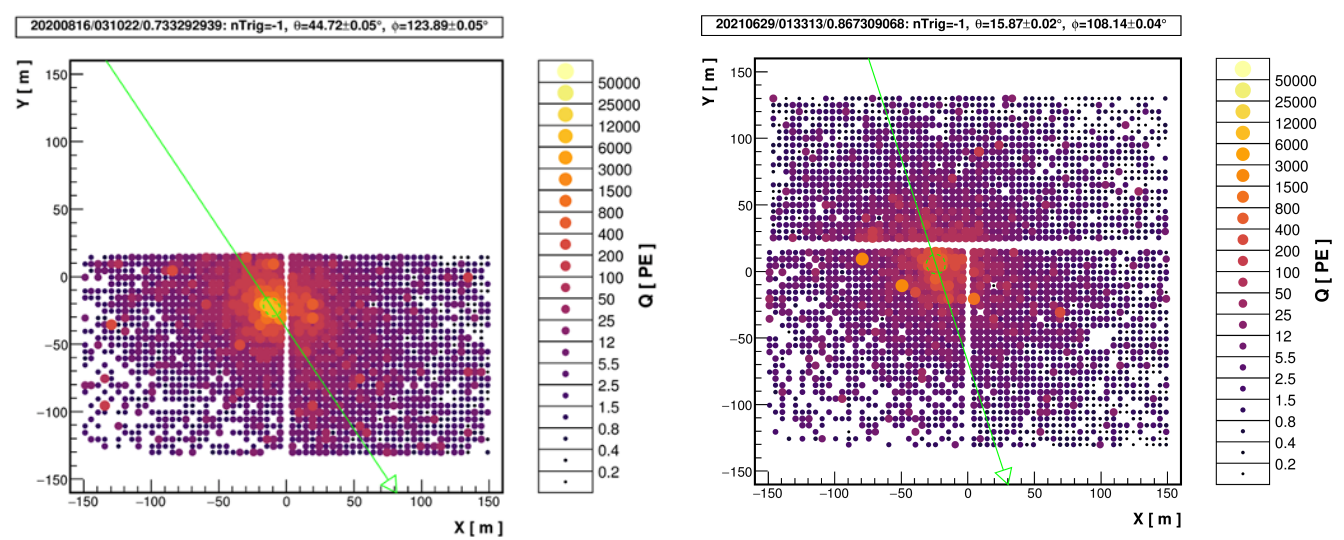

Figure 2: Left panel is a typical high energy event recored by WCDA-1 + WCDA-2 detectors, and right panel is also a high energy event detected by WCDA full array. Right color panel shows the number of photoelectrons, $\mathrm{N}_{P E}$, recorded by the $8 " / 20 "$ PMT in each cell.

WCDA has been built in 3 phases. The first one, denoted as WCDA-1, is a square pond of $150 \mathrm{~m} \times 150 \mathrm{~m}$ consisting of 900 detector units. The 900 detector units divide into 25 clusters and each cluster is comprised of 4 groups in which one group is made up of 9 detector units. One electronics board control 9 detectors units that are one group. Each detector unit is $5 \mathrm{~m} \times 5 \mathrm{~m}$ that includes one 1.5 inches PMT and one 8 inches PMT. It was completed in April 2019 and has been in operation since then. In the second phase, one more pond of the same size, referred to as WCDA-2, has also been in operation since March of 2020. In the thir phase, the third pond, WCDA-3, with a size of $300 \mathrm{~m} \times 110 \mathrm{~m}$, began operation at the March of 2021. For gamma-ray induced showers, WCDA-2 and WCDA-3 have a threshold below $100 \mathrm{GeV}$ because they use a 20" PMT and 3" PMT instead of (8" $\left.+1.5^{\prime \prime}\right)$ combination.

\section{Data and Analysis}

The events are reconstructed by first fitting the spatial charge distribution to identify the location of the primary particle trajectory as projected onto the array, which we refer to as the shower "core", and then fitting the arrival times of the hits with a conical shower front, the details about reconstruction and its angular resolution and shower core resolution can be find in the paper [3].

After air showers have been reconstructed, two different background estimation are used, one is called equal-zenith method [4], another is called direct integration [5]. The use of water cherenkov technique has been proven an effective technique to separate gamma-ray showers from the isotropic cosmic-ray background. After using mass sensitive parameter [5], a sky map with gamma-like events could be constructed.

The simulations consist of two steps, the first one is the air shower propagation simulation in the atmosphere which is handled by the CORSIKA package [6], another one is that the so-called G4WCDA programe based on the Geant4 [7] is used to simulate the detector response to secondary air shower particles. Using above simulation samples, a 


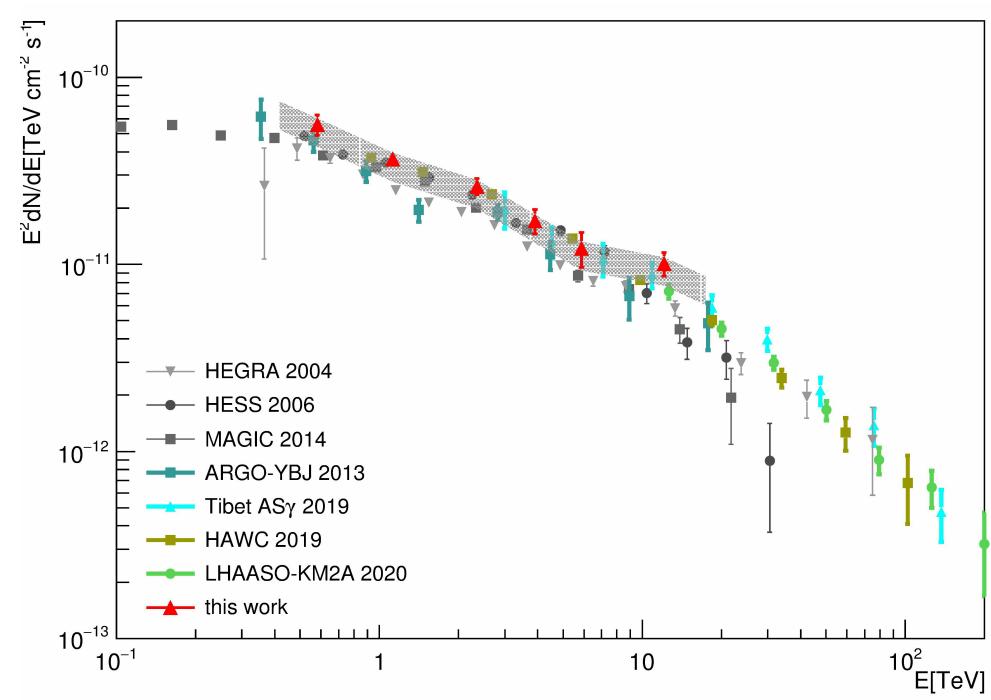

Figure 3: Top left panel is one month significance map of the Crab Nebular for events with $N_{\text {hits }}>170$, top right map is the distribution of excess events as function of the space angle with respect to the Crab Nebular center. Bottom panel is the spectral energy distribution of the Crab Nebula in the energy range from $800 \mathrm{GeV}$ to $13 \mathrm{TeV}$ measured by WCDA-1 one year data. As a comparison, the SED measured by using LHAASO-KM2A and other experiments are also plotted for comparison.

typical forward folding method is applied to estimate the spectrum energy distribution of the sources.

In this paper, we present results from two datasets:

- WCDA-1 single pool data from April. 2019 to Sep. of 2020. Roughly speaking, this dataset serverd as a function of a detector performance checking of our detector for the standard candle, the Crab Nebular observation.

- WCDA full array data, which is starting data-taking since Marc. 2021.

\section{Some selected results}

Firstly we present the observation of the standard candle Crab Nebular from WCDA-1 single pool. As effective fired number of PMTs, $N_{\text {hits }}$, higher than 170, which is relative to energy around $3 \mathrm{TeV}$, just as expected from the simulation, the significance of the Crab is higher than 20 sigma, and the sigma of $2 \mathrm{D}$ gaussian fitting of Crab image is around $0.2^{\circ}$, which means the angular resolution in this energy region is less than 0.4 deg. Fig. 3. is the spectral energy distribution of the Crab Nebular from $0.5 \mathrm{TeV}$ to $20 \mathrm{TeV}$. As a comparison, the SED measured by using LHAASO-KM2A[8] above $10 \mathrm{TeV}$ is also shown as the green filled circles. The result shows a good agreement with all other major gamma ray astronomy experiments. Such consistent results mean our simulation, analysis framework works correctly and effectively. 

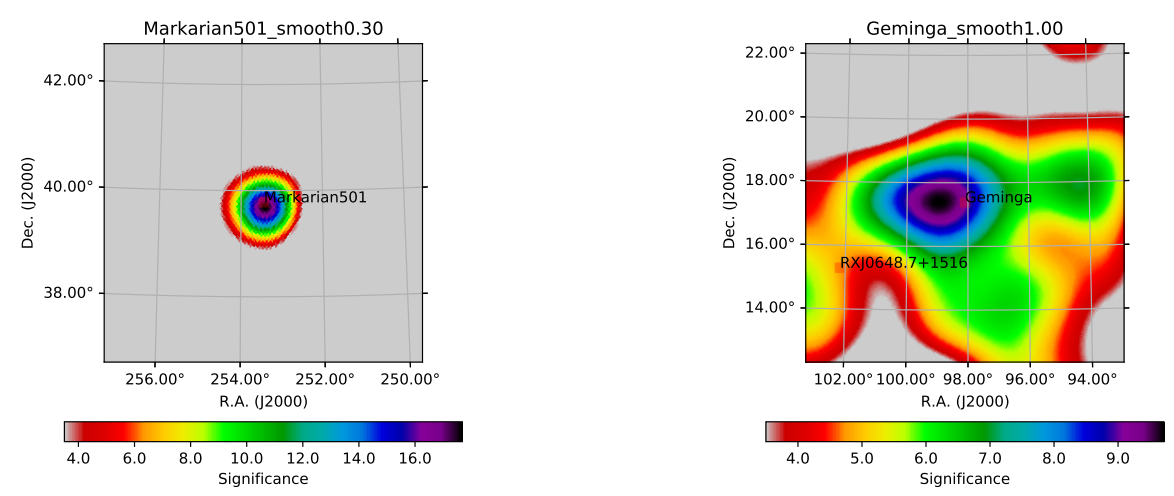

Figure 4: Sky map around Mrk-501 and Geminga by using WCDA full array data.

Since Mar. 2021, WCDA enters into full array data-taking mode. The famous active galactic nuclei Mrk 501 and well-nown Geminga region have been observed with the significance higher than 10 shown in Fig. 4 based on preliminary data analysis.

\section{Summary and Outlook}

WCDA full array has started scientific data-taking in the March of 2021. With its wide field of view, continuous operation and its good sensitivity, in the near future, some new results in $\mathrm{TeV}$ astronomy should be expected. At the same time, many efforts on the optmization of the Gamma/Proton separation, 8" and 20" PMT matching are still under studying.

\section{6. acknowledgments}

We would like to thank all staff members who work at the LHAASO site above 4400 meter above the sea level year round to maintain the detector and keep the water recycling system, electricity power supply and other components of the experiment operating smoothly. We are grateful to Chengdu Management Committee of Tianfu New Area for the constant financial support for research with LHAASO data. This research work is also supported by the following grants: The National Key R\&D program of China under grants 2018YFA0404201, 2018YFA0404202 and 2018YFA0404203, by the National Natural Science Foundation of China (NSFC grants No.12022502, No.11905227, No.U1931112, No.11635011, No.11761141001, No.Y811A35, No.11675187, No.U1831208, No.U1931111), and in Thailand by RTA6280002 from Thailand Science Research and Innovation.

\section{References}

[1] Z. Cao, Chin. Phys. C, 34 (2)(2010): 249-252;

[2] H. He (for LHAASO Coll.), Radiation Detection Technology and Methods 2-7, (2018) 
[3] LHAASO Collaboration Chinese Physics C Vol.45, No. 8(2021) 085002

[4] AS-Gamma Collaboration, ApJ, 525 (1999), L93-L96.

[5] MILAGRO Collaboration, APJ, 595 (2) (2003) 803-811.

[6] http://www-ik.fzk.de/corsika.

[7] S. Agostinelli, et al, Geant4 Collaboration, Nucl. Inst. Methods Phys. Res. A, 506(2003): 250-303.

[8] LHAASO Collaboration, Chinese Physics C Vol.45 (2021) 025002

LHAASO Collaboration Full Author List

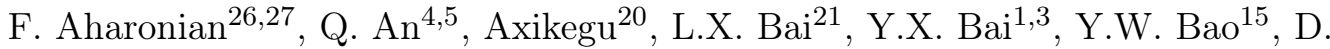
Bastieri $^{10}$, X.J. Bi ${ }^{1,2,3}$, Y.J. Bi ${ }^{1,3}$, H. Cai ${ }^{23}$, J.T. Cai ${ }^{10}$, Z. Cao ${ }^{1,2,3}$, Z. Cao ${ }^{4,5}$, J. Chang ${ }^{16}$, J.F. Chang ${ }^{1,3,4}$, X.C. Chang ${ }^{1,3}$, B.M. Chen ${ }^{13}$, J. Chen ${ }^{21}$, L. Chen ${ }^{1,2,3}$, L. Chen ${ }^{18}$, L. Chen $^{20}$, M.J. Chen ${ }^{1,3}$, M.L. Chen ${ }^{1,3,4}$, Q.H. Chen ${ }^{20}$, S.H. Chen ${ }^{1,2,3}$, S.Z. Chen ${ }^{1,3}$, T.L. Chen $^{22}$, X.L. Chen ${ }^{1,2,3}$, Y. Chen ${ }^{15}$, N. Cheng ${ }^{1,3}$, Y.D. Cheng ${ }^{1,3}$, S.W. Cui ${ }^{13}$, X.H. Cui ${ }^{7}$, Y.D. Cui ${ }^{11}$, B.Z. Dai ${ }^{24}$, H.L. Dai ${ }^{1,3,4}$, Z.G. Dai ${ }^{15}$, Danzengluobu ${ }^{22}$, D. della Volpe ${ }^{31}$, B.

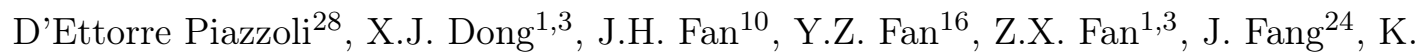
Fang $^{1,3}$, C.F. Feng ${ }^{17}$, L. Feng ${ }^{16}$, S.H. Feng ${ }^{1,3}$, Y.L. Feng ${ }^{16}$, B. Gao ${ }^{1,3}$, C.D. Gao ${ }^{17}$, Q.

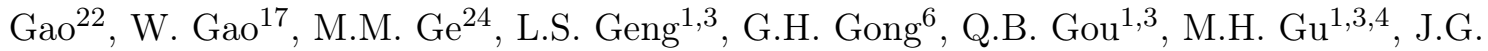

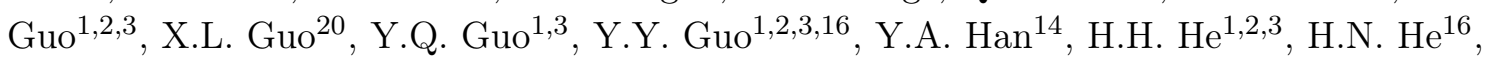
J.C. $\mathrm{He}^{1,2,3}$, S.L. $\mathrm{He}^{10}$, X.B. $\mathrm{He}^{11}$, Y. $\mathrm{He}^{20}$, M. Heller ${ }^{31}$, Y.K. Hor ${ }^{11}$, C. Hou ${ }^{1,3}$, X. Hou ${ }^{25}$,

H.B. $\mathrm{Hu}^{1,2,3}$, S. $\mathrm{Hu}^{21}$, S.C. Hu${ }^{1,2,3, \star}$, X.J. Hu${ }^{6}$, D.H. Huang ${ }^{20}$, Q.L. Huang ${ }^{1,3}$, W.H. Huang $^{17}$, X.T. Huang ${ }^{17}$, Z.C. Huang ${ }^{20}$, F. Ji ${ }^{1,3}$, X.L. Ji ${ }^{1,3,4}$, H.Y. Jia ${ }^{20}$, K. Jiang ${ }^{4,5}$, Z.J.

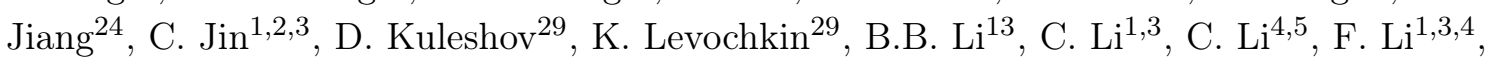
H.B. $\mathrm{Li}^{1,3}$, H.C. $\mathrm{Li}^{1,3}$, H.Y. Li ${ }^{5,16}$, J. Li ${ }^{1,3,4}$, K. Li ${ }^{1,3}$, W.L. $\mathrm{Li}^{17}$, X. Li ${ }^{4,5}$, X. Li ${ }^{20}$, X.R. $\mathrm{Li}^{1,3}$, Y. $\mathrm{Li}^{21}$, Y.Z. $\mathrm{Li}^{1,2,3}$, Z. $\mathrm{Li}^{1,3}$, Z. Li ${ }^{9}$, E.W. Liang ${ }^{12}$, Y.F. Liang ${ }^{12}$, S.J. Lin ${ }^{11, \star}$, B. $\mathrm{Liu}^{5}$, C. $\mathrm{Liu}^{1,3}$, D. $\mathrm{Liu}^{17}$, H. Liu ${ }^{20}$, H.D. Liu ${ }^{14}$, J. Liu ${ }^{1,3}$, J.L. Liu ${ }^{19}$, J.S. Liu ${ }^{11}$, J.Y. Liu ${ }^{1,3}$,

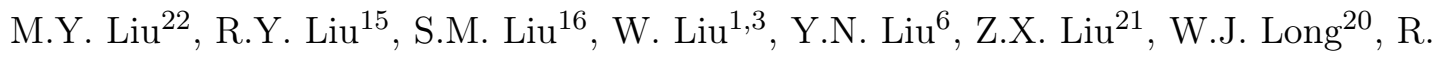

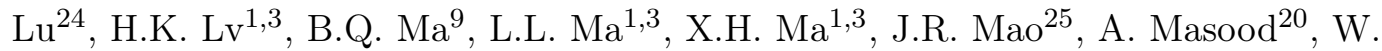
Mitthumsiri $^{32}$, T. Montaruli ${ }^{31}$, Y.C. Nan ${ }^{17}$, B.Y. Pang ${ }^{20}$, P. Pattarakijwanich ${ }^{32}$, Z.Y. $\mathrm{Pei}^{10}$, M.Y. Qi ${ }^{1,3}$, B.Q. Qiao ${ }^{1}$, D. Ruffolo ${ }^{32}$, V. Rulev ${ }^{29}$, A. Sáiz ${ }^{32}$, L. Shao ${ }^{13}$, O. Shchegolev $^{29,30}$, X.D. Sheng ${ }^{1,3}$, J.R. Shi ${ }^{1,3}$, H.C. Song ${ }^{9}$, Yu.V. Stenkin ${ }^{29,30}$, V. Stepanov ${ }^{29}$, Q.N. $\operatorname{Sun}^{20}$, X.N. Sun ${ }^{12}$, Z.B. $\operatorname{Sun}^{8}$, P.H.T. Tam ${ }^{11}$, Z.B. Tang ${ }^{4,5}$, W.W. Tian ${ }^{2,7}$, B.D.

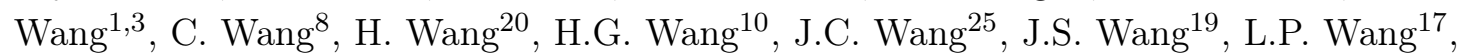

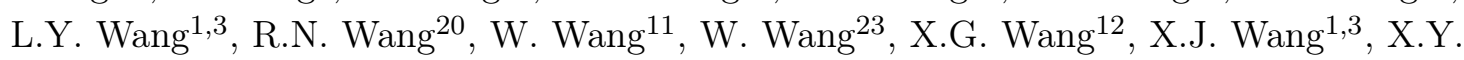

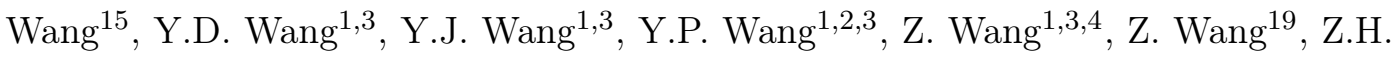
Wang $^{21}$, Z.X. Wang ${ }^{24}$, D.M. Wei ${ }^{16}$, J.J. Wei ${ }^{16}$, Y.J. Wei ${ }^{1,2,3}$, T. Wen ${ }^{24}$, C.Y. Wu ${ }^{1,3}$, H.R. $\mathrm{Wu}^{1,3, \star}$, S. $\mathrm{Wu}^{1,3}$, W.X. $\mathrm{Wu}^{20}$, X.F. $\mathrm{Wu}^{16}$, S.Q. Xi ${ }^{20}$, J. Xia ${ }^{5,16}$, J.J. Xia ${ }^{20}$, G.M.

$\mathrm{Xiang}^{2,18, \star}$, G. Xiao ${ }^{1,3}$, H.B. Xiao ${ }^{10}$, G.G. Xin ${ }^{23}$, Y.L. Xin ${ }^{20}$, Y. Xing ${ }^{18}$, D.L. Xu ${ }^{19}$, R.X.

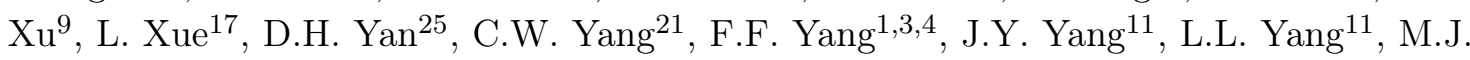

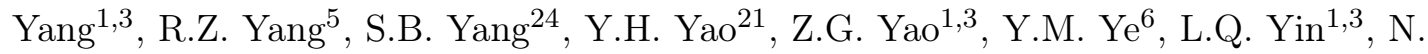
$\mathrm{Yin}^{17}$, X.H. You ${ }^{1,3}$, Z.Y. You ${ }^{1,2,3}$, Y.H. Yu ${ }^{17}$, Q. Yuan ${ }^{16}$, H.D. Zeng ${ }^{16}$, T.X. Zeng ${ }^{1,3,4}$, W. 
Zeng $^{24}$, Z.K. Zeng ${ }^{1,2,3}$, M. Zha ${ }^{1,3, \star}$, X.X. Zhai ${ }^{1,3}$, B.B. Zhang ${ }^{15}$, H.M. Zhang ${ }^{15}$, H.Y. Zhang $^{17}$, J.L. Zhang ${ }^{7}$, J.W. Zhang ${ }^{21}$, L. Zhang ${ }^{13}$, L. Zhang ${ }^{24}$, L.X. Zhang ${ }^{10}$, P.F. Zhang $^{24}$, P.P. Zhang ${ }^{13}$, R. Zhang ${ }^{5,16}$, S.R. Zhang ${ }^{13}$, S.S. Zhang ${ }^{1,3}$, X. Zhang ${ }^{15}$, X.P. Zhang $^{1,3}$, Y. Zhang ${ }^{1,3}$, Y. Zhang ${ }^{1,16}$, Y.F. Zhang ${ }^{20}$, Y.L. Zhang ${ }^{1,3}$, B. Zhao ${ }^{20}$, J. Zhao ${ }^{1,3}$, L. Zhao ${ }^{4,5}$, L.Z. Zhao ${ }^{13}$, S.P. Zhao ${ }^{16,17}$, F. Zheng ${ }^{8}$, Y. Zheng ${ }^{20}$, B. Zhou ${ }^{1,3}$, H. Zhou ${ }^{19}$, J.N. Zhou ${ }^{18}$, P. Zhou ${ }^{15}$, R. Zhou ${ }^{21}$, X.X. Zhou ${ }^{20}$, C.G. Zhu ${ }^{17}$, F.R. Zhu ${ }^{20}$, H. Zhu ${ }^{7}$, K.J. $\mathrm{Zhu}^{1,2,3,4}, \mathrm{X} . \mathrm{Zuo}^{1,3}$

(The LHAASO Collaboration)

${ }^{1}$ Key Laboratory of Particle Astrophyics \& Experimental Physics Division \& Computing Center, Institute of High Energy Physics, Chinese Academy of Sciences, 100049 Beijing, China

${ }^{2}$ University of Chinese Academy of Sciences, 100049 Beijing, China

${ }^{3}$ TIANFU Cosmic Ray Research Center, Chengdu, Sichuan, China

${ }^{4}$ State Key Laboratory of Particle Detection and Electronics, China

${ }^{5}$ University of Science and Technology of China, 230026 Hefei, Anhui, China

${ }^{6}$ Department of Engineering Physics, Tsinghua University, 100084 Beijing, China

${ }^{7}$ National Astronomical Observatories, Chinese Academy of Sciences, 100101 Beijing, China

${ }^{8}$ National Space Science Center, Chinese Academy of Sciences, 100190 Beijing, China

${ }^{9}$ School of Physics, Peking University, 100871 Beijing, China

${ }^{10}$ Center for Astrophysics, Guangzhou University, 510006 Guangzhou, Guangdong, China

${ }^{11}$ School of Physics and Astronomy \& School of Physics (Guangzhou), Sun Yat-sen University, 519082 Zhuhai, Guangdong, China

${ }^{12}$ School of Physical Science and Technology, Guangxi University, 530004 Nanning, Guangxi, China

${ }^{13}$ Hebei Normal University, 050024 Shijiazhuang, Hebei, China

${ }^{14}$ School of Physics and Microelectronics, Zhengzhou University, 450001 Zhengzhou, Henan, China

${ }^{15}$ School of Astronomy and Space Science, Nanjing University, 210023 Nanjing, Jiangsu, China

${ }^{16}$ Key Laboratory of Dark Matter and Space Astronomy, Purple Mountain Observatory,

Chinese Academy of Sciences, 210023 Nanjing, Jiangsu, China

${ }^{17}$ Institute of Frontier and Interdisciplinary Science, Shandong University, 266237 Qingdao, Shandong, China

${ }^{18}$ Key Laboratory for Research in Galaxies and Cosmology, Shanghai Astronomical

Observatory, Chinese Academy of Sciences, 200030 Shanghai, China

${ }^{19}$ Tsung-Dao Lee Institute \& School of Physics and Astronomy, Shanghai Jiao Tong University, 200240 Shanghai, China

${ }^{20}$ School of Physical Science and Technology \& School of Information Science and Technology, Southwest Jiaotong University, 610031 Chengdu, Sichuan, China ${ }^{21}$ College of Physics, Sichuan University, 610065 Chengdu, Sichuan, China

${ }^{22}$ Key Laboratory of Cosmic Rays (Tibet University), Ministry of Education, 850000 Lhasa, Tibet, China 
${ }^{23}$ School of Physics and Technology, Wuhan University, 430072 Wuhan, Hubei, China ${ }^{24}$ School of Physics and Astronomy, Yunnan University, 650091 Kunming, Yunnan, China ${ }^{25}$ Yunnan Observatories, Chinese Academy of Sciences, 650216 Kunming, Yunnan, China ${ }^{26}$ Dublin Institute for Advanced Studies, 31 Fitzwilliam Place, 2 Dublin, Ireland ${ }^{27}$ Max-Planck-Institut for Nuclear Physics, P.O. Box 103980, 69029 Heidelberg, Germany 28 Dipartimento di Fisica dell'Università di Napoli "Federico II", Complesso Universitario di Monte Sant'Angelo, via Cinthia, 80126 Napoli, Italy.

${ }^{29}$ Institute for Nuclear Research of Russian Academy of Sciences, 117312 Moscow, Russia ${ }^{30}$ Moscow Institute of Physics and Technology, 141700 Moscow, Russia

${ }^{31}$ Département de Physique Nucléaire et Corpusculaire, Faculté de Sciences, Université de Genève, 24 Quai Ernest Ansermet, 1211 Geneva, Switzerland

${ }^{32}$ Department of Physics, Faculty of Science, Mahidol University, 10400 Bangkok, Thailand 